\title{
El paisaje como infraestructura. Caso de estudio: el río Salí en el sistema metropolitano de Tucumán (SIMET)
}

\author{
María Paula Llomparte*
}

\section{Resumen}

La incorporación de la noción de paisaje como infraestructura se presenta como una alternativa estratégica para la generación de un espacio participativo, de calidad ambiental y de inclusión social, que atienda a recursos culturales y naturales en el marco de la planificación del territorio.

Las dinámicas y procesos de expansión del Área Metropolitana de Tucumán (AMeT) han afectado al río Salí como recurso y a sus paisajes como componentes fundamentales para la concreción de un modelo más cercano a la sustentabilidad. Desde mediados del siglo XX hasta la actualidad se impulsaron numerosos planes y propuestas que procuran revertir esta situación.

El presente trabajo propone revisar las diferentes propuestas de actuación en los márgenes del Salí a los fines de evaluar si propician o atienden al paisaje como elemento estructurante del espacio metropolitano. Se argumenta que los planes propuestos se reproducen desde una lógica netamente extractivista, afectando al río como patrimonio cultural y ambiental y desatendiendo el aprovechamiento responsable de los recursos territoriales.

\footnotetext{
* Arquitecta. Becaria Postgrado Tipo I CONICET. Observatorio de Fenómenos Urbanos Territoriales, Cátedra de Urbanismo I, Instituto de Planeamiento y Desarrollo Urbano, FAU, UNT.
} 


\section{Palabras clave}

Paisaje - infraestructura territorial - río Salí.

\section{Abstract}

The incorporation of the notion of landscape as infrastructure is presented as a strategic option for the generation of a space for participation, environmental quality, and social inclusion that is responsive to cultural and natural resources within the framework of the planning of the territory.

The dynamics and processes of expansion of the Metropolitan Area of Tucumán (amet) have affected the Salí river as a resource and its landscapes as key components for the realization of a model closer to sustainability. Since the midtwentieth century until the present numbers prompted plans and proposals that seek to reverse this situation.

This paper proposes to revise the various proposals for action for the purposes of assessing whether these favor or take care of the landscape as a structuring element of metropolitan space. It is argued that the proposed plans are reproduced from a purely logical extractive affecting to the river, such as environmental and cultural heritage, and disregard the responsible utilization of territorial resources.

\section{Keywords}

Landscape - territorial infrastructure - Sali river.

\section{Introducción}

El continuo crecimiento de las ciudades, los diferentes tipos de espacios (casiespacios virtuales) y las nuevas estructuras sociales (sociedad hipertexto) requieren intervenciones flexibles en las que participen las nuevas tecnologías y formas de entender los servicios públicos ${ }^{1}$, una especie de ciudad à la carte como plantea Ascher (Óp. cit.).

Diversos autores aluden al término de revolución urbana o tercera revolución urbana. Según Borja (2007) esta etapa se caracteriza por: a) las modificación relaciones tiempo espacio a través de la informatización y nuevas redes internet telefonía móvil, b) la

\footnotetext{
${ }^{1}$ Ascher propone un cambio en el concepto de infraestructura y su financiación en relación con el urbanismo moderno, en el cual se atribuía a lo público la responsabilidad de las grandes infraestructuras y equipamientos colectivos. En el neourbanismo las infraestructuras y equipamientos entremezclan intervenciones públicas y privadas.
} 
expansión urbana discontinua con centralidades diversas, c) gobernabilidades débiles y fragmentadas en virtud de estos nuevos territorios urbano-regionales y $d$ ) el retiro del estado benefactor (welfare state), encargado de la "reproducción social" (vivienda, educación, servicios sociales, etc.).

En América Latina, los procesos de expansión se caracterizan por la difusión urbana fragmentada, la pérdida de potencial social, entendido como derecho a ciudad y a un espacio de calidad ambiental, reduciendo los sistemas naturales de alto valor paisajístico a territorios vacantes según presiones y lógicas de apropiación territorial que degradan la calidad del entorno y del paisaje, componentes fundamentales para la concreción de un modelo más cercano a la sustentabilidad.

El paisaje como instrumento de gestión territorial es un componente reciente en la Argentina y en particular para la provincia de Tucumán. El presente trabajo propone una reflexión sobre las diversas situaciones de producción y estrategias de apropiación del entorno fluvial del río Salí en las últimas décadas, en correspondencia con el AMeT.

Desde estas miradas se analizarán las propuestas de actuación en el río Salí, buscando identificar si incluyen alternativas novedosas que consideren al paisaje como infraestructura o, por el contrario, se realizan según lógicas de construcción del territorio más tradicionales.

\section{La gestión del paisaje}

El término paisaje, en su concepción más amplia como el legado de la experiencia y el esfuerzo de la comunidad, fuera material o inmaterial, surge a principios de siglo XX. Carl Sauer (1967) expone que el campo de la geografía es el paisaje, considerado como la división primaria del conocimiento. El paisaje es definido como el área compuesta por una asociación distintiva de formas, tanto físicas como culturales, conteniendo por un lado por el sitio — suma de todos los recursos naturales que el ser humano tiene a su disposición en esa área-y por otro lado su expresión cultural.

En las últimas décadas, ante la amenaza de intervenciones poco respetuosas con la coherencia del paisaje, se formularon numerosos planes y documentos. En el año 2000, en Florencia, Los Estados Miembros del Consejo de Europa celebran el Convenio Europeo del Paisaje (CEP), a través del cual se asume "la importancia del paisaje para la formación de las culturas locales y que es un componente fundamental del patrimonio natural y cultural, que contribuye al bienestar de los seres humanos y a la consolidación de la identidad europea".

Este convenio tiene numerosos aspectos positivos. M. Frolova (2009) rescata en primer lugar que convierte al paisaje en un objeto de derecho de las poblaciones. En segundo lugar, continúa, "no se limita sólo a una consideración de los paisajes excepcionales, sino que concierne igualmente a aquellos que constituyen el marco de la 
vida cotidiana e, incluso, a los que necesitan una reestructuración, abarcando todo el conjunto del territorio europeo".

En el contexto norteamericano emerge la noción de Landscape Urbanism, haciendo referencia al paisaje como medio de ordenación urbana para la ciudad contemporánea, centrándose especialmente en las crecientes infraestructuras que son necesarias para su adecuado funcionamiento. El Urbanismo Paisajista es producto de dos corrientes. Por un lado, una corriente convencional dentro de la arquitectura y ecología paisajistas en el ámbito del urbanismo regional. Por otro lado, la postura más radical, la más ecologista, apuesta por las medidas de los años sesenta y setenta, que abogaban por unos métodos urbanísticos alejados de los principios de la ingeniería (C. Waldheim, 2006). Los impulsores de este movimiento reivindican las aproximaciones de Olmsted, Geddes, Mumford o Ian McHarg, y su punto de partida es que el paisaje es la fuerza generadora de las metrópolis contemporáneas.

En Latinoamérica se cuenta con Cartas del paisaje, instrumento de concertación de estrategias entre los agentes públicos y privados, con el fin de llevar a cabo actuaciones de reconocimiento, valoración, protección, gestión y ordenación del paisaje. Ejemplo de ellas son las de México, Colombia, Brasil y Argentina, entre otras. Otro avance en lo referente al paisaje es la creación de la Red Latinoamericana de Paisaje por las Universidades de Argentina, Brasil y México.

Por su lado, la Red Argentina del Paisaje plantea que

más allá de los valores estéticos del paisaje, está aquel paisaje cotidiano o aquel paisaje degradado por el uso, por la especulación inmobiliaria o como fruto de la exclusión social. Es por ese paisaje por donde la población transita, no importa su condición, su género o su edad.

Además, en el marco de la red se cuenta con una Subcomisión Nacional de Observatorios y Cartas del Paisaje y la Subcomisión Nacional sobre Ley de Protección, Gestión y Ordenamiento del Paisaje, cuyo objetivo es la construcción de los Observatorios Regionales del Paisaje y la sanción de la Ley de Presupuestos Mínimos sobre Paisaje.

En función de las diferentes realidades sociales, el paisaje se puede configurar como un espacio visible o como un espacio marginado, olvidado. En este sentido, es resultado de la potente acumulación de hechos y mecanismos que subyacen a las representaciones socioculturales.

\section{Metodología}

Las propuestas de planes sobre los márgenes y el curso del río Salí se analizan mediante un estudio descriptivo y explicativo. Estas tareas se realizaron a través de la recopilación de fuentes secundarias de información (antecedentes bibliográficos, 
cuadros y mapas referenciales, informes técnicos institucionales) y herramientas visuales (fotografías, cartografía, imágenes satelitales).

Se comparan las escalas de abordaje, impactos esperados y resultados concretos de cada propuesta.

Para la espacialización de los datos y realización de mapas se utilizó el software gratuito gvSIG 11.

\section{Caracterización del área de estudio}

La provincia de Tucumán, ubicada al norte de la República Argentina, posee una población de 1.448 .200 habitantes, en una superficie de $22.524 \mathrm{~km}^{2}$ (INDEC, censo 2010). La alta densidad de su población se distribuye en el espacio de manera no homogénea, concentrándose más del 70 \% en el Área Metropolitana de Tucumán $(\mathrm{AMeT}){ }^{2}$ tendiendo a un crecimiento mayor, como se manifiesta hace ya varias décadas.

En la configuración del espacio metropolitano coexisten áreas urbanas, donde las características del medio natural fueron muy modificadas por un sistema antrópico de tipo urbano en pleno proceso expansivo, y áreas rurales de uso agrícola intensivo. A ellas se suman espacios naturales (Sierra San Javier y cuenca del Salí) que por sus valores ecológicos y su biodiversidad configuran un soporte natural del que depende en gran medida el equilibrio de la aglomeración metropolitana. (PNUMA, 2007)

El río Salí forma parte de la cuenca Salí-Dulce, cuyas nacientes están en Cumbres Calchaquíes, Sierra de Santa Bárbara, provincia de Salta; atraviesa el territorio tucumano con dirección norte-sur aproximadamente hasta que llega a la ciudad San Miguel de Tucumán. Al sur de esta cambia la dirección al SE para llegar al Embalse El Frontal en la vecina provincia santiagueña. Una vez que ingresa en la provincia de Santiago del Estero, recibe el nombre de Dulce. La cuenca forma parte de las provincias de Salta, Tucumán, Santiago del Estero y Córdoba.

La importancia ecológica de la cuenca se debe a que el río Salí es el principal colector fluvial de la provincia: en él desemboca el $80 \%$ de los ríos y arroyos provenientes de las estribaciones montañosas. Butí y Cancino (2005) señalan que además de constituir la principal fuente de agua para consumo humano, ganado y riego, el río Salí es importante por su heterogeneidad geográfica y los servicios ecosistémicos que brinda a un vasto territorio.

\footnotetext{
${ }^{2}$ Integran el espacio metropolitano los municipios de San Miguel de Tucumán (ciudad cabecera), Yerba Buena, Banda del Río Salí, Tafi Viejo, Las Talitas, Alderetes y seis comunas rurales.
} 
A estos valores se suman su relevancia histórica como actor decisivo del traslado de San Miguel de Tucumán desde Ibatín en 1685 hasta su actual emplazamiento en "La Toma". El traslado de la ciudad se realiza en forma literal, la planta urbana de Ibatín se repite sin variantes en el nuevo lugar. Pero incluso antes de la llegada española, sus márgenes ya contaban con asentamientos prehispánicos, de los cuales se cuenta con evidencia arqueológica según el antropólogo Caria (2006).

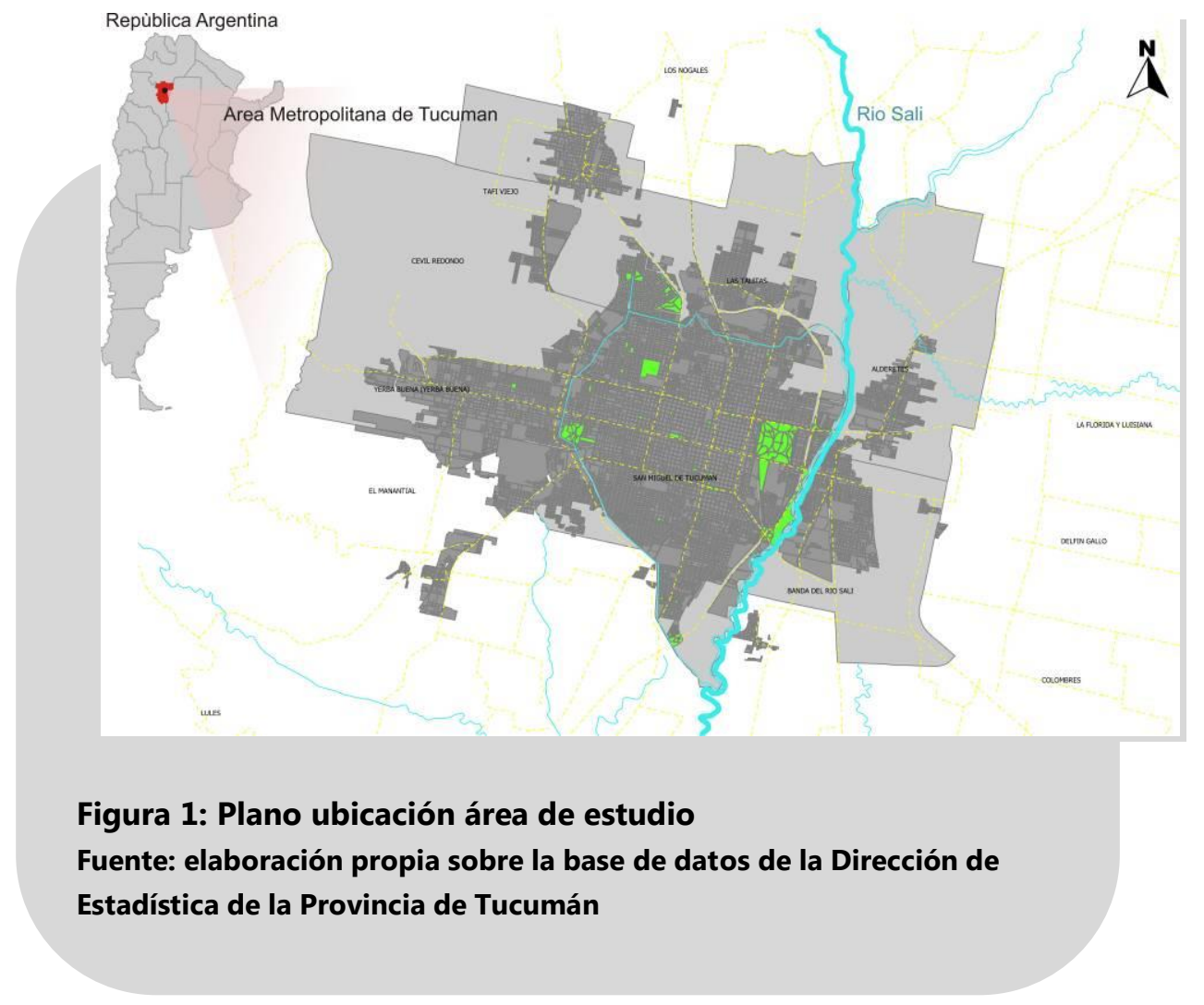

A mediados de la década de 1960 se inicia el proceso de metropolización de Tucumán a través de la consolidación de núcleos poblacionales suburbanos que hoy configuran la conurbanización del Gran San Miguel de Tucumán. El crecimiento posterior de la ciudad de San Miguel de Tucumán hasta nuestros días se produce mediante dos tendencias, hacia el oeste, marcada por la segregación social fomentada principalmente por iniciativas privadas como barrios cerrados, countries, y hacia el este, más heterogénea, con fuerte incidencia del Estado como promotor de vivienda y la presencia de asentamientos informales.

Esta situación genera un área periurbana espacial y socialmente fragmentada, producto principalmente de la carencia de una estrategia coordinada de desarrollo y de la heterogeneidad de los agentes involucrados. Por otro lado, el proceso sociohistórico de urbanización del Gran San Miguel de Tucumán agudiza esta segregación: mientras 
el cerro San Javier es un espacio visible, altamente calificado paisajística y ambientalmente, el río Salí es un espacio marginal, invisible y de borde.

El río Salí quedó inmerso en medio de esta manera de construcción de suelo urbano, difuso y disperso, sin lineamiento claros de actuación. Esta situación produjo graves condiciones de deterioro en su curso, por vertidos contaminantes en su cauce (de origen industrial y líquidos cloacales sin tratamiento) y disposición de residuos sólidos urbanos. Además, se suman la presencia de asentamientos irregulares en sus bordes con grandes déficit de infraestructura básica (cloacas, gas, agua potable) y la intensiva actividad minera en sus bordes (extracción de áridos).

Otra problemática que presenta el curso del río es la cantidad de actores (Nación, Provincia y Municipios) que tiene injerencia en su curso. La gestión de cuencas hídricas es administrada por el Estado, que debe ejercer controles sobre la totalidad de las fuentes de agua, dictando y haciendo cumplir la normativa para el aprovechamiento y protección de las diversas fuentes de agua como una sola fuente de suministro.

Particularmente, la provincia de Tucumán se encuentra dividida en departamentos, municipios y comunas rurales. Los departamentos carecen de función administrativa, y actualmente su finalidad está ligada exclusivamente al Régimen Electoral. La Constitución Provincial, reformada en 2006, ha incorporado la autonomía municipal; en consecuencia, la administración y la gestión del territorio corresponden a los municipios, actuando las comunas rurales como delegaciones territoriales del gobierno provincial.

A raíz de las problemáticas generadas entre la provincia de Tucumán y Santiago del Estero por el tema de la contaminación del cauce del río, generadas en el SIMeT y luego recibidas en el embalse de Río Hondo, ya jurisdicción de Santiago del Estero, se plantea la necesidad de la creación de un comité. En el marco de la Ley Nacional N. ${ }^{\circ}$ 25688, de Presupuestos Mínimos para la Gestión Ambiental de las Aguas, según lo dispuesto en el Artículo $4{ }^{3}$ se crea en el Comité Interjurisdiccional de la Cuenca del río Salí Dulce.

\section{Antecedentes: planes y propuestas en relación con el río Salí}

En la revisión de antecedentes, la primera propuesta que incorpora una mirada crítica y lineamientos de actuación a la situación del río es el "Estudio del Plan Regulador del Municipio de San Miguel De Tucumán", realizado en 1956 con horizonte a 1986, por el arquitecto Cino Calcaprima.

\footnotetext{
3 Artículo 4: Créanse, para las cuencas interjurisdiccionales, los comités de cuencas hídricas con la misión de asesorar a la autoridad competente en materia de recursos hídricos y colaborar en la gestión ambientalmente sustentable de las cuencas hídricas.
} 
En el planteo están presentes ideas propias del racionalismo, como la zonificación de actividades y la jerarquización de vías de circulación. Es así como para la circulación interurbana y suburbana propone la creación de una avenida Costanera (actual avenida de Circunvalación), que además de soportar el tránsito pesado de carácter interregional, procedente de Salta por la ruta 9, tendrá una función de esparcimiento e instalaciones deportivas.

También se plantean áreas de reforestación con plantas de eucaliptos de una larga faja que comprenderá la superficie recuperada al río y que se destinará a reserva pública de bosque y parque. En el año 1956 ya se describe en el plan la existencia de ranchos de extrema pobreza en los márgenes del río; estos pertenecían a personas que trabajaban de la explotación de la arena. Ante esta situación, se propone la eliminación y rehabilitación de las viviendas antihigiénicas.

Respecto de la zonificación de actividades, la existencia de zonas industriales en los márgenes del río Salí, según el plan, no se puede ignorar, ya que es la que posiciona a nuestra ciudad a escala regional; por lo tanto, se aconseja la previsión de zonas industriales en zonas aledañas a la ciudad, destinadas a desarrollarse en el tiempo.

En cuanto a zonas de verdes y de esparcimiento, plantea la instalación en el límite este de la ciudad de un núcleo deportivo de alcance regional, lindante con el río, además de las zonas reforestadas propuestas anteriormente.

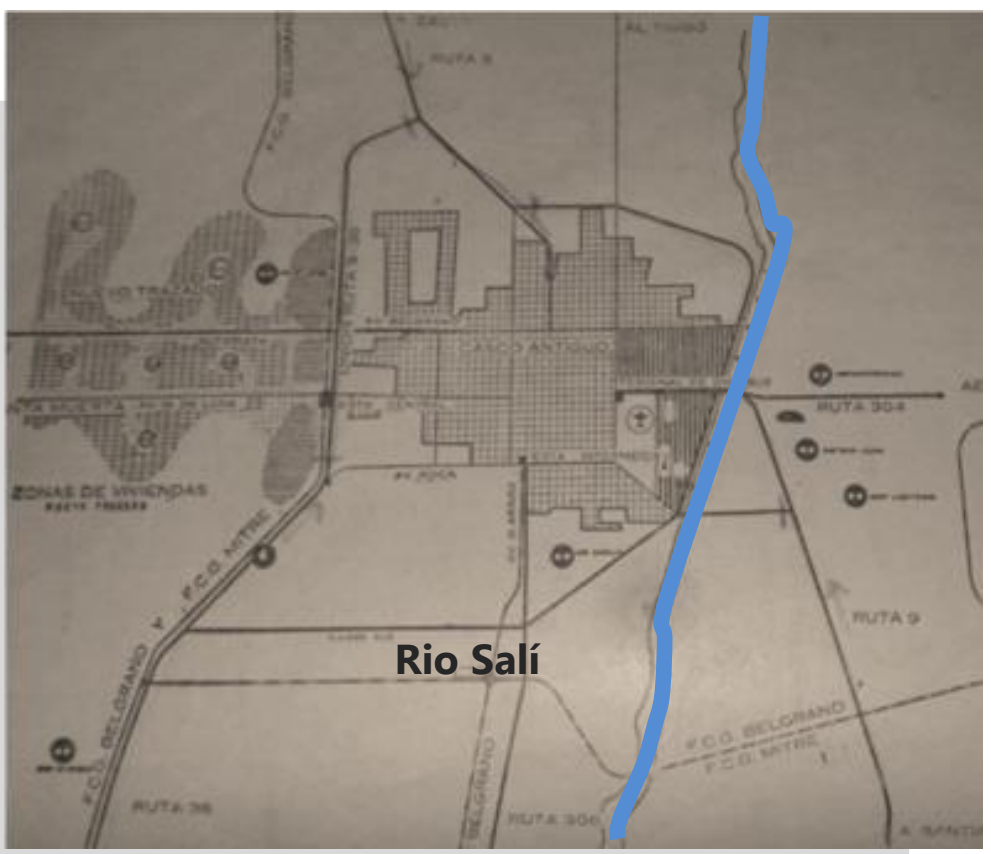

Figura 2: planteo del Plan Regulador Fuente: estudio del Plan regulador del municipio de San Miguel de Tucumán 1956-1986. 


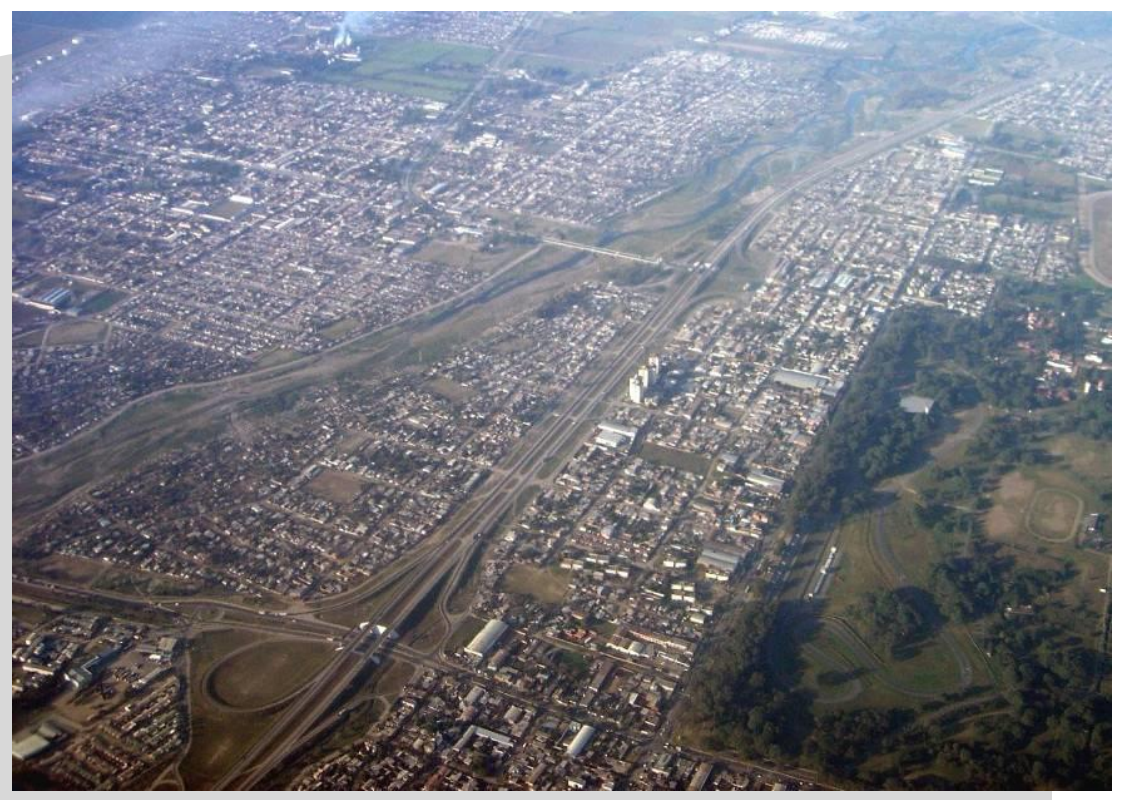

Figura 3: Vista área de avenida de Circunvalación Fuente: http://www.panoramio.com/photo/12700718

El río Salí vuelve a tener protagonismo en la agenda publica recién en el año 2005, mediante el Plan Estratégico de San Miguel de Tucumán 2016, en el que se plantea entre sus principales líneas estratégicas la recuperación paisajística y ambiental del Salí. Esta línea de actuación tiene la finalidad de poner en relación todos aquellos proyectos que contemplen la ejecución de obras tendientes a mejorar las condiciones ambientales y paisajísticas del territorio, a través de la concreción del corredor este (margen correspondiente al municipio capitalino). La recualificación del sector se realizará a partir del saneamiento ambiental, la sistematización del curso de agua y la reubicación de la población que vive en los bordes del río en situación de riesgo. Los proyectos específicos que se proponen para este "corredor ambiental" son la creación de un paseo ribereño, la rectificación del curso de agua, el control de efluentes cloacales y la creación de un parque regional con áreas deportiva y recreativa. Se propone, además, alentar los proyectos que tengan por objetivos recuperar, mejorar y poner en valor el paisaje, de modo tal que San Miguel de Tucumán vuelva a ser el Jardín de la República. 


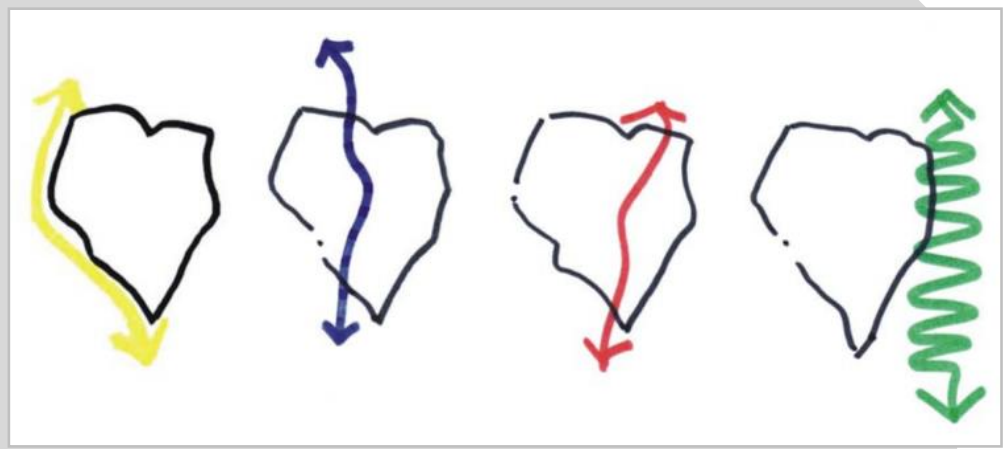

Figura 4: Estrategia de intervención por corredores estructurales norte-sur.

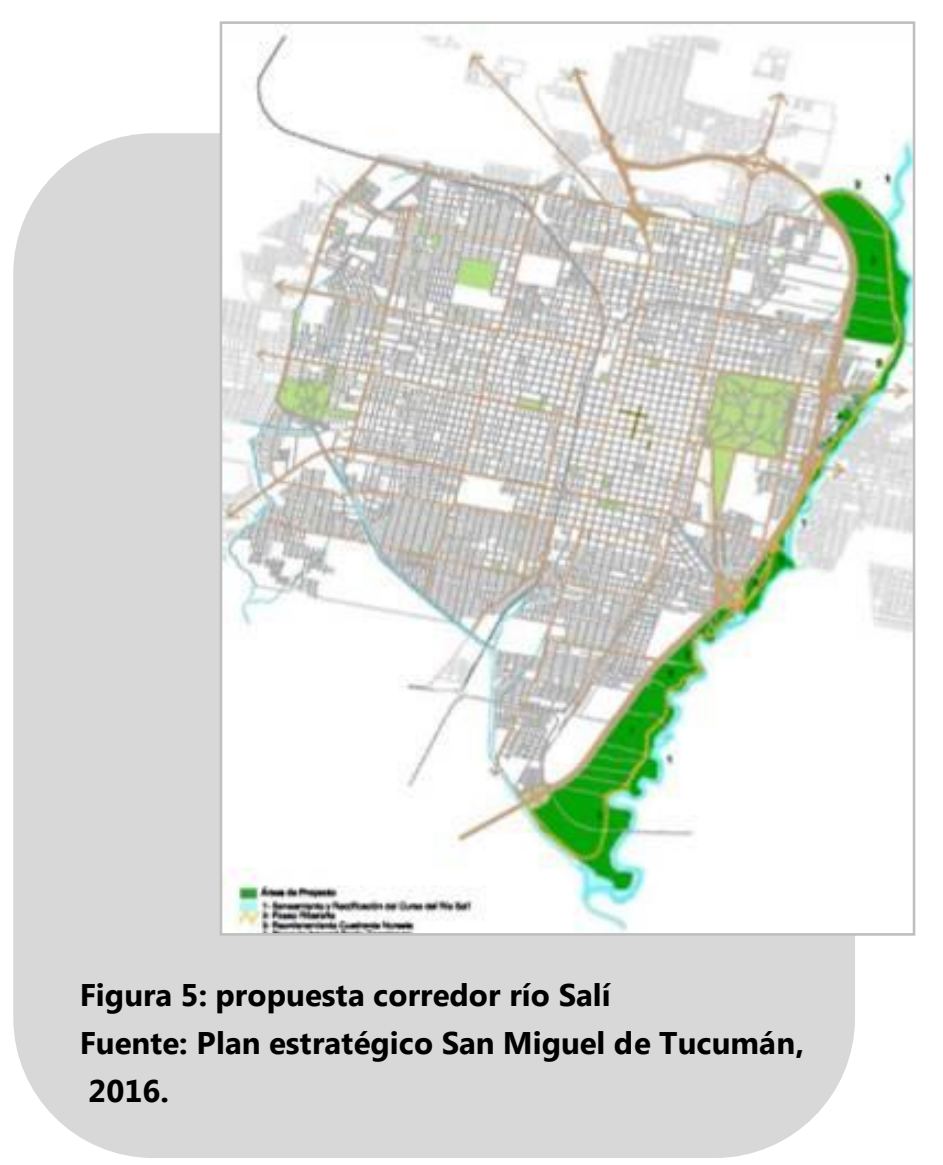

A escala regional, se cuenta con el Plan de gestión de la cuenca, formulado en 2006 por la Secretaría de Ambiente y Desarrollo Sustentable de la Nación, la Subsecretaría de Recursos Hídricos y los gobiernos de Santiago del Estero y Tucumán. Tiene como objetivo alcanzar una mejora sustancial en la calidad de vida de la población de la 
cuenca, asegurando el desarrollo de las actividades productivas, en un marco de equidad y de sustentabilidad de los aspectos ambientales involucrados. En consonancia con el Plan de Gestión de la Cuenca se confecciona el Acuerdo Marco para un Plan de reconversión industrial en la provincia de Tucumán, en el cual se propone desarrollar y ejecutar un plan para las industrias situadas en el ámbito de la Cuenca del Salí Dulce para el enfoque preventivo y la adopción de mecanismos y políticas para el desarrollo industrial sustentable, destinados al fortalecimiento, competitividad y desarrollo del sector industrial.

En el año 2009 se licitó el Plan maestro para la recuperación y desarrollo de la costanera del río Sali en la ciudad de San Miguel de Tucumán. Su objetivo es formular proyectos para la intervención urbana en los espacios de la ribera correspondiente solamente a la ciudad de San Miguel de Tucumán, en el marco de los avances producidos con el Plan Estratégico. Estas tareas suponen articular la ciudad a través de proyectos que incluyan actividades recreativas, productivas y sociales. La propuesta de configuración espacial se basa en la creación de sistemas de espacios verdes de distintas categorías, metropolitano, intermedios, corredores verdes que se suman a los espacios verdes ya existentes; un sistema hidráulico que incluye la canalización del río y sistemas de vínculos a través de la estructura vial (primaria, secundaria y transporte medioambiental circular) y de la estructura de usos territoriales. Los proyectos se concentran en la realización de un parque metropolitano, un parque central y en la generación de sectores residenciales y de industrias.

Actualmente se están llevando a cabo en el marco de este plan las obras de canalización del curso y el Programa Mejoramiento de Barrios (PROMEBA) en el Municipio San Miguel de Tucumán. Las obras propuestas en el marco del PROMEBA II buscan ser un aporte directo para la inclusión urbana y social de las familias que viven en los barrios lindantes al cauce del río. La intervención del Programa de Mejoramiento de Barrios (PROMEBA), ejecutado con el apoyo de la Subsecretaría de Vivienda del Ministerio de Planificación Federal, Inversión Pública y Servicios, La Unidad Coordinadora Nacional (UCN), la Unidad Ejecutora Provincial (UEP) y promovido desde el Municipio San Miguel de Tucumán, tiene como propósito mejorar de modo sustentable el hábitat de los hogares residentes en asentamientos irregulares, con intervenciones orientadas a la provisión de infraestructura, equipamiento público, saneamiento ambiental y legalización de la tenencia de las tierras. 

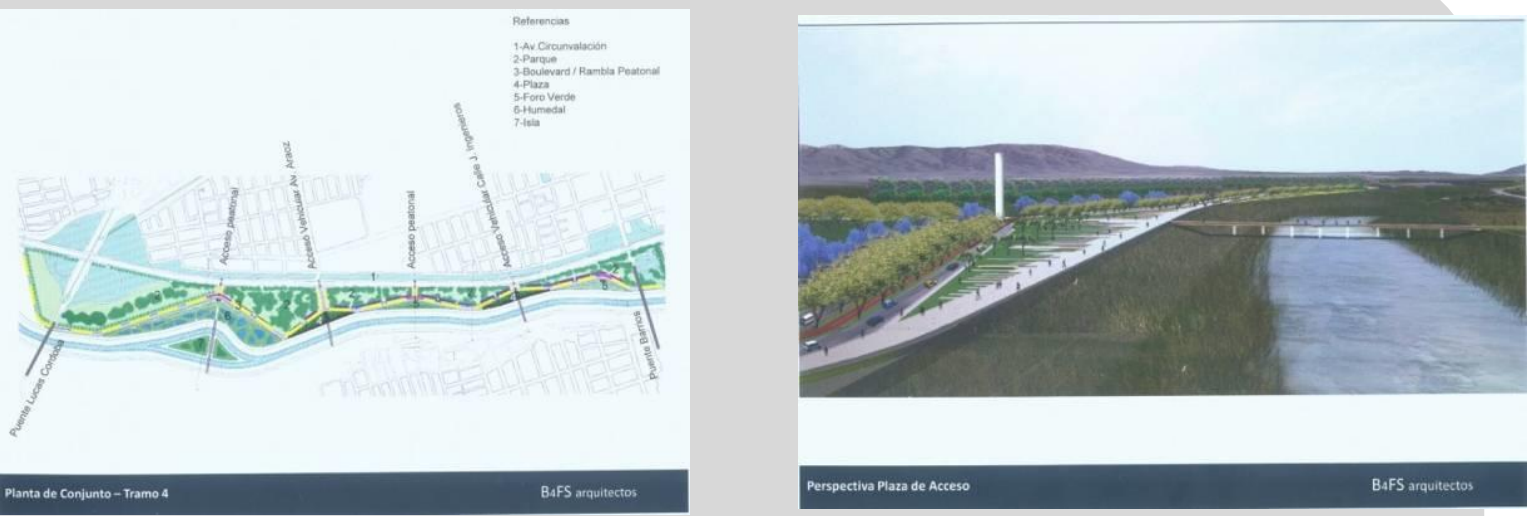

Gráfico 5: propuesta plan maestro para el margen río Salí S. M. de Tucumán

Fuente: disponible web. http://www.skyscrapercity.com/showthread.php?p=73252827
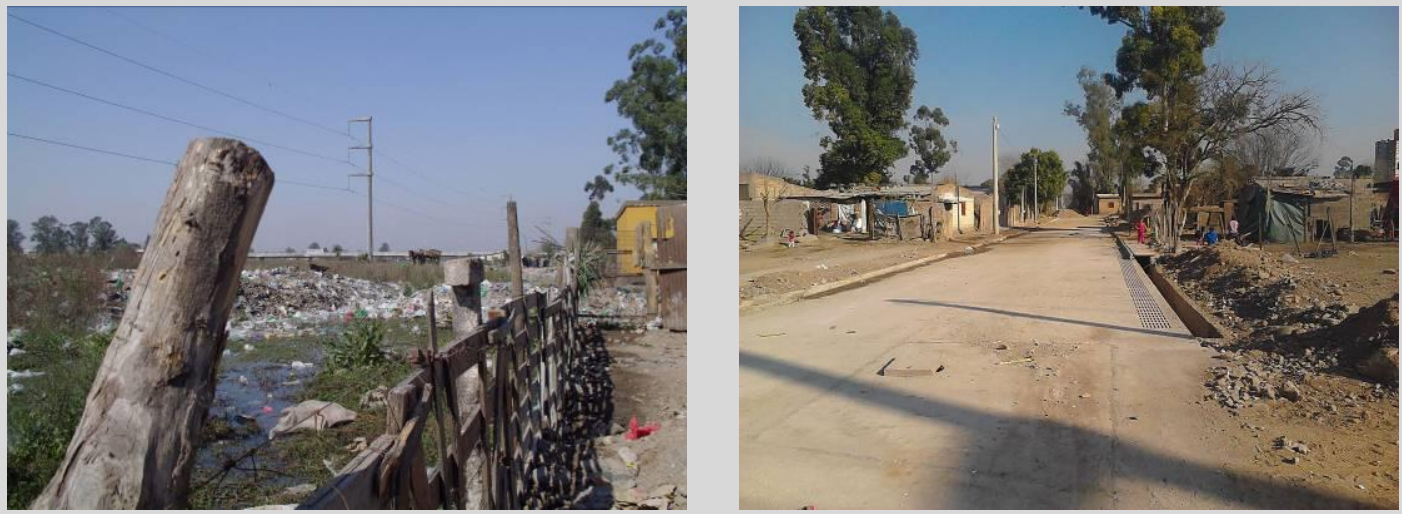

Gráfico 6: situación costanera antes y después

Fuente: Instituto Provincial de la Vivienda Tucumán

Gráfico 7: cuadro comparativo intervenciones

\begin{tabular}{|c|c|c|c|c|}
\hline PLAN & $\begin{array}{c}\text { AÑO DE } \\
\text { FORMULACIÓN }\end{array}$ & ESCALA & PROPUESTA & $\begin{array}{l}\text { ACTUACIONES } \\
\text { CONCRETAS }\end{array}$ \\
\hline Estudio & 1956 & Municipi & 1. Avenida & Avenida \\
\hline $\begin{array}{l}\text { del Plan } \\
\text { Regulador } \\
\text { del } \\
\text { Municipio }\end{array}$ & & $\begin{array}{l}\text { O de } \\
\text { Tucumán }\end{array}$ & $\begin{array}{l}\text { Costanera (actual } \\
\text { avenida de } \\
\text { Circunvalación) } \\
\text { 2. Áreas de }\end{array}$ & Circunvalación \\
\hline
\end{tabular}




\begin{tabular}{|c|c|c|c|c|}
\hline $\begin{array}{l}\text { de San } \\
\text { Miguel de } \\
\text { Tucumán }\end{array}$ & & & $\begin{array}{l}\text { grandes } \\
\text { equipamientos } \\
\text { 3. Áreas de } \\
\text { reforestación } \\
5 . \\
\text { Sistematización } \\
\text { del cauce }\end{array}$ & \\
\hline \begin{tabular}{l}
\multicolumn{2}{c}{ Plan } \\
Estratégico \\
de SMT \\
2016
\end{tabular} & 2005 & $\begin{array}{l}\text { Municipi } \\
\text { o de } \\
\text { Tucumán }\end{array}$ & $\begin{array}{l}\text { 1. Corredores } \\
\text { estratégicos norte- } \\
\text { sur }\end{array}$ & $\begin{array}{l}\text { Se concretaron } \\
\text { algunas } \\
\text { propuestas } \\
\text { específicas en } \\
\text { otros corredores; } \\
\text { sin avances en el } \\
\text { corredor Salí. }\end{array}$ \\
\hline $\begin{array}{l}\text { Plan de } \\
\text { gestión de la } \\
\text { cuenca }\end{array}$ & 2006 & $\begin{array}{l}\text { Regional } \\
\text { Provincia } \\
\text { de } \\
\text { Tucumán, } \\
\text { Córdoba, } \\
\text { Santiago } \\
\text { del Estero }\end{array}$ & $\begin{array}{l}\text { 1.Reducción de } \\
\text { la contaminación } \\
\text { 2. Control de la } \\
\text { erosión y la } \\
\text { sedimentación } \\
\text { 3. Recuperación de } \\
\text { embalses y } \\
\text { preservación de } \\
\text { bañados y } \\
\text { lagunas } \\
\text { 4. Prevención } \\
\text { de sequías e } \\
\text { inundaciones }\end{array}$ & \begin{tabular}{l}
\multicolumn{2}{c}{ Se avanzó en el } \\
Plan de \\
reconversión \\
industrial en la \\
provincia \\
Tucumán
\end{tabular} \\
\hline \begin{tabular}{l}
\multicolumn{1}{c}{ Plan } \\
maestro \\
para la \\
recuperación \\
y desarrollo \\
de la \\
costanera \\
del río Salí
\end{tabular} & 2009 & $\begin{array}{l}\text { Municipi } \\
0 \quad \text { de } \\
\text { Tucumán }\end{array}$ & $\begin{array}{l}\text { 1. Sistemas de } \\
\text { espacios verdes, } \\
\text { 2. Sistema } \\
\text { hidráulico que } \\
\text { incluye la } \\
\text { canalización del } \\
\text { río } \\
\text { 3. Sistemas de } \\
\text { vínculos a través } \\
\text { de la estructura } \\
\text { vial y de la de usos } \\
\text { territoriales. }\end{array}$ & $\begin{array}{l}\text { Sector } \\
\text { residencia, } \\
\text { consolidación } \\
\text { asentamiento } \\
\text { informal Programa } \\
\text { PROMEBA. }\end{array}$ \\
\hline
\end{tabular}




\section{Discusiones}

Los planes y propuestas sobre la ribera del Salí ponen de manifiesto las problemáticas prioritarias que hay que atender en cuanto a mejorar condiciones de calidad de vida para la población residente en la cuenca y al saneamiento de las aguas, pero este proceso es incipiente. Se avanzó con respuestas puntales, principalmente en el Municipio de San Miguel de Tucumán.

Las propuestas de actuación sobre el río Salí no logran romper la lógica tradicional de producción de territorio, priorizando aspectos pertinentes al propio metabolismo urbano por sobre los requerimientos del recurso natural, en este caso el río Salí.

Se continúa imponiendo una zonificación de actividades de acuerdo con la trama existente, donde se concentran las actividades en determinados sectores perdiendo las mixturas propias del habitar el paisaje. La materialización formal de una costanera mediante la canalización del río descuida valores ambientales, sociales y culturales de suma importancia.

La falta de armonía en las relaciones sociedad-naturaleza permanece en las propuestas y en la sociedad en general. La apropiación del entorno natural por parte de la sociedad implica un hecho político que lleva impresas las contradicciones existentes entre la cultura y el entorno natural. La dimensión del paisaje busca identificar estas relaciones hacia una comprensión de la lógica de cómo se produce territorio para la generación de alternativas de gestión que contemplen los activos locales como promotores del desarrollo.

Estas actuaciones ponen de manifiesto la falta de una cultura del agua (que entiende al recurso hídrico como patrimonio) y una percepción del espacio litoral como paisaje por parte de los diferentes actores sociales. La Nueva Cultura del Agua ha incorporado el enfoque del CEP y todas sus potencialidades para un planteamiento global de los valores y de los problemas del agua (Mata Olmo y Fernández Muñoz, 2010). Los autores plantean que las relaciones entre agua y paisaje han estado ausentes hasta ahora en las políticas públicas, que de modo específico o indirectamente se han venido ocupando de la gestión de los recursos hídricos.

Aun persiste en la práctica la mirada tradicional de infraestructura planteada desde una visión netamente económica que excluye al paisaje como elemento fundamental. Malelisz (1963) desarrolló su Teoría de los Umbrales Urbanos, en la cual referencia cómo el crecimiento espacial de los pueblos tropieza con limitaciones físicas de carácter topográfico o debidas a las características tecnológicas de los diversos servicios de utilidad pública. La inversión necesaria para incorporar al uso urbano un terreno determinado puede ser definida gráficamente mediante una curva de costo por habitante. Las inflexiones del gráfico van señalando las limitaciones al desarrollo en términos de ese costo. 
Esta mirada se evidencia en los planes expuestos, en los cuales la revitalización del río Salí recae en términos netamente formales, priorizando al proyecto urbanístico como catalizador de una imagen más acorde con una realidad global que con la local.

Por otro lado, no se cuenta con un plan a escala del sistema metropolitano que atienda al río Salí en todo su paso por el conglomerado urbano. Si bien los diversos planes tratan de incorporar la dimensión metropolitana en sus propuestas, esto no es viable en función de que financian obras para el municipio contratante, en este caso casi siempre la ciudad cabecera del área metropolitana, San Miguel de Tucumán. La otra margen del río es olvidada en la medida en que no se generen alianzas para subsanar esta situación.

Se deben pensar el río y su entorno como infraestructura metropolitana, sistema articulador y configurador del territorio. Las acciones en sus márgenes deben superar límites administrativos y promover el desarrollo desde la preservación de los recursos naturales y culturales como base del bienestar de la sociedad.

El desafío queda planteado en articular acciones entre sector privado / público y sociedad, sustentado en principios de cohesión social, gobernanza y participación, lo que implica una tarea de movilización, acuerdo, consenso entre actores, tendiente a alcanzar un desarrollo sostenible.

\section{BIBLIOGRAFÍA}

AQUINO, A., COLLANTES, M. Y MON, R (2006) "Desafíos ambientales del Gran San Miguel de Tucumán, Argentina". Facultad de Ciencias naturales e IML, Universidad Nacional de Tucumán. Edición Magna.

ASCHER, F. (2004). Los nuevos principios del urbanismo. Madrid: Alianza Ensayo.

BORJA, J. (2007). Revolución y contrarrevolución en la ciudad global: las expectativas frustradas por la globalización de nuestras ciudades. Revista EURE. Santiago de Chile Vol. XXXIII, N. ${ }^{\circ} 100$, pp. 35-50.

CAMINOS, R.; BOMBA, H.; CASARES, M. y DI LULLO, R. (2007). GEO San Miguel de Tucumán, Perspectivas del Medio Ambiente. Tucumán.

CENTRO DE ESTUDIO AMBIENTALES (2012). El Anillo Verde Interior. Hacia una Infraestructura Verde Urbana en Vitoria-Gasteiz. Vitoria-Gasteiz.

CONVENIO EUROPEO DEL PAISAJE (2000). Aprobado por el Consejo de Europa y marco de referencia europeo para el diseño de políticas de protección, gestión y ordenación del paisaje en toda Europa.

FROLOVA, M. (2009) "La evolución reciente de las políticas de paisaje en España y el convenio europeo del paisaje". Revista Proyección N. ${ }^{\circ}$ 6, [en línea], Instituto CIFOT, Universidad nacional de Cuyo, Argentina. Disponible en Internet: http://www.proyeccion.cifot.com.ar/?page id $=449$ 
NOGUÉ, J. et ál. (2012). Franges. Els paisatges de la periferia. Barcelona: Editorial Observatorio del paisaje de Cataluña. (Plecs de paisatge: Reflexions 3).

NOGUĖ, J. (ed.) (2007). La construcción social del paisaje. Madrid: Colección Paisaje y teoría. Editorial Biblioteca.

MATA OLMO, R. Y FERNÁNDEZ MUÑOZ, S. (2010) "Paisajes y patrimonios culturales del agua", Panel Científico-Técnico de Seguimiento de La Política de Aguas, Sevilla, España.

MUNICIPALIDAD DE SAN MIGUEL DE TUCUMÁN (1956). Estudio del plan regulador del municipio de San Miguel de Tucumán 1956-1986. Tucumán.

MUNICIPALIDAD DE SAN MIGUEL DE TUCUMÁN (2005). Plan estratégico Urbano Territorial para la Ciudad de San Miguel de Tucumán. Tucumán.

MUNICIPALIDAD DE SAN MIGUEL DE TUCUMÁN (2009). Plan Maestro para la recuperación y desarrollo de la costanera del Río Salí de la ciudad de San Miguel de Tucumán. Tucumán.

SECRETARÍA DE MEDIO AMBIENTE Y DESARROLLO SUSTENTABLE DE LA NACIÓN (2006). Cuenca del Río Salí Dulce. Documento base del Plan de Gestión.

RICCI, T. R. (1967). Evolución de la ciudad de San Miguel de Tucumán. Tucumán: Ediciones Sesquicentenarias de la Independencia Argentina. Universidad Nacional de Tucumán.

WALDHEIM, CHARLES (2006) "Paisaje como infraestructura". Conferencia Junta Andalucía, España. $\quad\{E n \quad$ línea\} http://www.opandalucia.es/index.php?form $=15 \&$ accion $=213 \&$ userid $=25 \&$ groupid $=0$ 